\title{
Die pleistozäne Vereisung und ihre isostatischen Auswirkungen im Bereich des Bellsunds (West-Spitzbergen)
}

\author{
Von Gerhard Stäblein, Würzburg
}

Mit 5 Abbildungen

\begin{abstract}
$\mathrm{Z}$ us a m menfassung. Aufgrund der morphologischen Formanalyse ist eine pleistozäne Eisbedeckung im Bellsund-Bereich von durchschnittlich 400-800 m nachzuweisen. Das Ansteigen der Schliffgrenze nach $\mathrm{E}$ und die nach $\mathrm{E}$ führenden Taltröge weisen eine hochglaziale Eisüberfließung von E her nach. Eine zweite tiefere Schliffgrenze aus dem Spätglazial wird am Fjordausgang durch einen westvergenten markanten Schliffbord bei rd. $120 \mathrm{~m}$ deutlich.

Die heutige Vergletscherung entspricht Gletschervorstößen nach der postglazialen Wärmezeit. Dabei haben die kleineren Talgletscher im Stand vom Ende des 19. Jh. ihre maximale holozäne Ausdehnung erreicht. Nur die größeren Fjordendgletscher zeigen weit vorgeschobene Moränen aus gestauchten, holozänen, marinen Sedimenten. Die Gletscher des Untersuchungsbereichs sind gegenüber der Kartierung von 1936 fast alle zurückgewichen.

Fossile Strandlinien holozänen Alters reichen sicher bis $60 \mathrm{~m}$, wahrscheinlich bis $85 \mathrm{~m}$ über das heutige Meeresniveau. Höhere Hangstufen bis $264 \mathrm{~m}$ sind Struktur-Terrassen mit Konglomeratgeröllstreu. Die Landhebung wird aufgefaßt als die elasto-plastische Reaktion der Erdkruste auf die quartäre Eisbelastung und Eisentlastung, die einer älteren Hebungstendenz aufgeprägt ist. Die besonders breite $20-\mathrm{m}$-Terrasse dürfte einer Phase des Ausgleichs von glazial-isostatischer Landhebung und glazial-eustatischem Meeresspiegelanstieg entsprechen. $\mathrm{Da}$ die marinen Terrassen im Bereich der $70 \mathrm{~km}$ langen Fjorde keine Verkippung zeigen, ist die Landhebung hier anders als im Ostteil Spitzbergens, nicht parallel zur nach W abnehmenden Eisbelastung, sondern im Block erfolgt.
\end{abstract}

Die regionalen Ergebnisse werden mit bisherigen Ergebnissen aus anderen Gebieten Spitzbergens verglichen.

$\mathrm{Su} \mathrm{m} \mathrm{m} \mathrm{a} \mathrm{ry.} \mathrm{The} \mathrm{pleistocene} \mathrm{ice-sheet} \mathrm{of} \mathrm{c.} \mathrm{400-800} \mathrm{m}$ in the Bellsund-area can be proved with the geomorphological analyses. The ascending of the ice-scour limit eastwards and the trough-valleys pointing to $\mathrm{E}$ prove a movement of ice-sheet from the $\mathrm{E}$ in the main-glacial period. At the exit of the fjord a second, lower ice-scour limit can be seen at about $120 \mathrm{~m}$ as a well marked ice-scour terrace inclined to W from the late-glacial time.

The present glaciation corresponds to glacier-advances after the post-glacial warm-period. The glaciers of the small valleys had their maximal holocene extent in the positions of the end of the 19th century. Only the bigger glaciers at the end of the fjords show large and far-reaching moraines built of compressed holocene marine sediments. The glaciers in the field of research have nearly all retreated compared to maps of 1936.

The fossil shore-lines of holocene age certainly reach till $60 \mathrm{~m}$, probably till $85 \mathrm{~m}$ above present sea-level. Higher slopesteps till $264 \mathrm{~m}$ are structure-terraces with a spread of conglomerat-gravels. The uplift of the land is comprehended as an elasto-plastic reaction of the earth crust to the quartarnary ice-burden and ice-relieve impressed on an older tendency of uplift. The especially broad $20 \mathrm{~m}$-terrace corresponds probably to a phase of compensation of the glacial-isostatic uplift of land and the glacial-eustatic uplift of sea-level. As the marine terraces are not tilted in the $70 \mathrm{~km}$ long area of the fjords, the uplift of the land occurred there in block, not parallel to the ice-burden, which deminished to the W, differently to the eastern parts of Spitsbergen.

The regional results are compared with results from other regions of Spitsbergen.

Im Sommer 1968 wurde im Rahmen einer Expedition unter anderem die Frage nach der pleistozänen Vereisung und deren holozänen isostatischen Auswirkungen im Bereich des Bellsunds auf West-Spitzbergen untersucht. Die glaziologisch-geomorphologische Expedition wurde von Dr. GLASER vom Geographischen Institut der Universität Würzburg geleitet. Die Untersuchungen schlossen an die Forschungen an, die unter Leitung von Prof. BüDEL besonders in SE-Spitzbergen in den Jahren 1959, 1960 und 1967 durchgeführt wurden (BüDEL 1960, 1962). 


\section{Das Arbeitsgebiet}

Das Arbeitsgebiet gehört zum alpine Reliefzüge aufweisenden, fjordreichen Westteil Spitzbergens, der z. T. von einem ausgedehnten Eisstromnetz überzogen ist. Spitzbergen liegt zwischen 76 und $80^{\circ} \mathrm{n}$. Br. Von der Nordküste sind es noch $\mathrm{rd}$. $1000 \mathrm{~km}$ bis zum Nordpol.

Zwischen dem Eis-Fjord im $\mathrm{N}$ und dem Horn-Sund im S liegt der Bell-Sund, von dem aus der Van-Mijen-Fjord, der Van-Keulen-Fjord und der Recherche-Fjord ausgreifen. Auf diesen Bereich beziehen sich unsere Beobachtungen und Aussagen (Abb. 1).

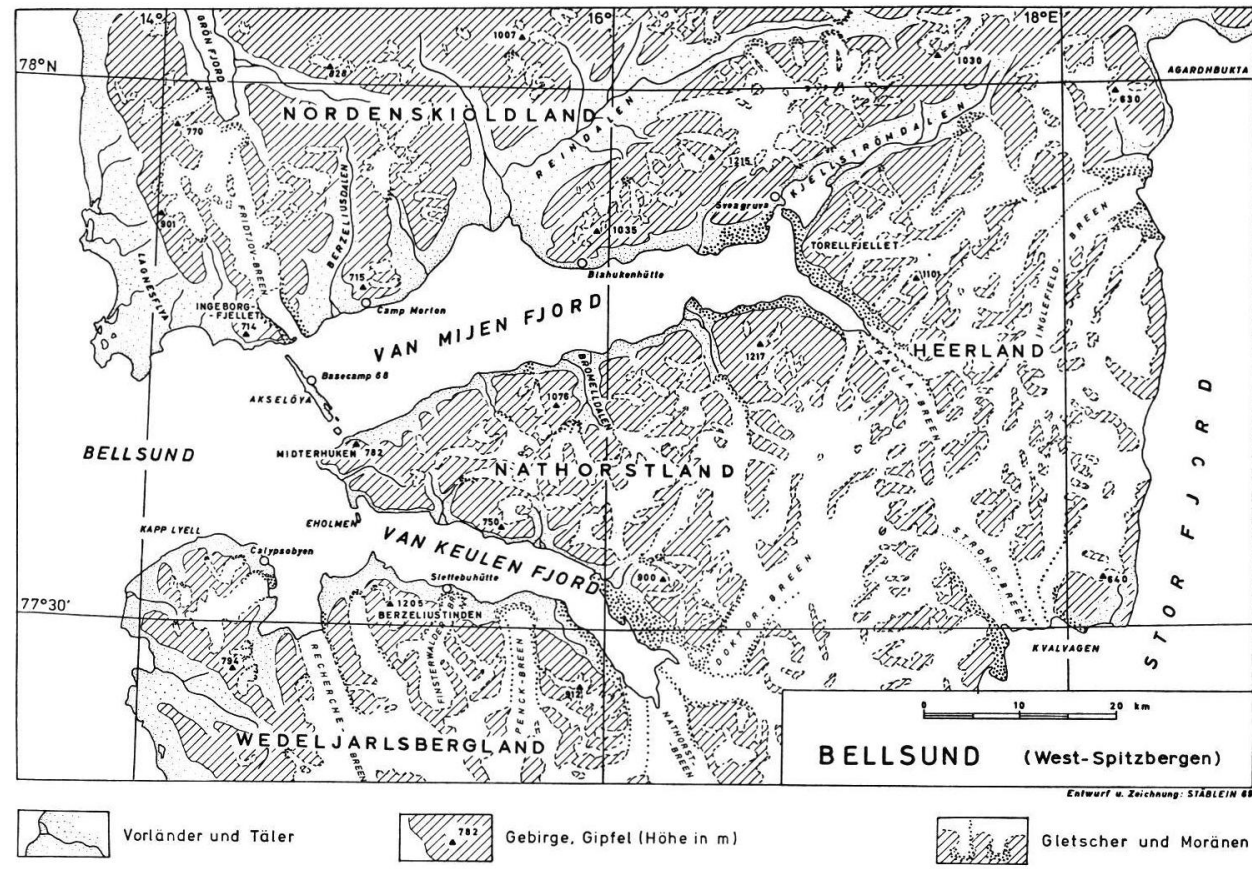

Abb. 1. Karte des Arbeitsgebietes, der Bellsundbereich in West-Spitzbergen.

\section{Forschungsstand}

Im Eis-Fjord, wo die wichtigen Kohlebergbausiedlungen liegen, das norwegische Longyearbyen und das russische Barentsburg, wurden die Strandterrassen von FeYLINGHanssen (1965) bearbeitet. Der Bereich des Horn-Sunds wurde von polnischen Expeditionen untersucht (JAHN 1959).

Im Bellsund-Bereich greift der Van-Mijen-Fjord $70 \mathrm{~km}$ tief ins Land ein, so daß ein Anschluß und Vergleich mit den Untersuchungsergebnissen SE-Spitzbergens von BüDEL (1968) aus dem Freeman-Sund-Bereich und von GLASER (1968) aus dem Stor-Fjord-Bereich möglich sind.

\section{Vorzeitformen}

Trotz der energischen heutigen Formbildungsdynamik der exzessiven Talbildungszone, die in diesem Frostschutt- und Tundrenbereich wirksam ist, wurden die Großformen im Landschaftsbild doch in erster Linie durch Formungsprozesse geprägt, die unter anderen klimatischen Bedingungen in den verschiedenen Vorzeiten abliefen.

Während des Pleistozäns von vor rd. 1 Million Jahre bis vor 10000 Jahren hat die Erde mehrmals Klimaverschlechterungen durch Rückgang der Temperaturen erlebt, durch 
die die Vergletscherungszonen besonders der arktischen Polarkalotte stark zunahmen. So war auch der Spitzbergen-Archipel in der Eiszeit von einer ausgedehnten Eismasse bedeckt. Mächtigkeit, Ausdehnung und Abfließrichtung des Eises der letzten Vereisung lassen sich bestimmen aus Spuren, denen die gemorphologische Formanalyse nachforscht. Gletschergeschliffene Vorzeitformen (Taltröge, Rundhöcker, Gletscherschliffe) und altc Moränen, z. T. weit über bzw. entfernt von den heutigen Gletscherrändern, sind eindeutige Beweise der größeren pleistozänen Vereisung Spitzbergens.

\section{Die heutigen Gletscher}

Die heutigen Gletscher sind nicht etwa der Überrest oder ein Rückzugsstadium der pleistozänen Vereisung, sondern im wesentlichen eine Neuvergletscherung nach der postglazialen Wärmezeit ( 9400 bis 2400 Jahre vor heute, nach FEYLING-Hanssen 1965). An vielen Stellen wurden nacheiszeitliche holozäne Sedimente, die offensichtlich in eisfreiem Gebiet abgelagert wurden, von den holozänen Gletschervorstößen überfahren und gestaucht. Durch den Gletscherrückgang der letzten Jahrzehnte wurden an mehreren Stellen solche holozäne überfahrene Sedimente sichtbar, die beweisen, daß die Vergletscherung im Holozän, in der Nacheiszeit, schon einmal wesentlich geringer gewesen sein muß.

Die Gletscher des Bellsund-Bereichs sind - mit Ausnahme des Recherche-Breen, der leicht vorgestoßen ist - alle z. T. sogar um km-Beträge, gegenüber der Kartenaufnahme von 1936 zurückgewichen.

Die klimatische Schneegrenze liegt heute bei rd. $600 \mathrm{~m}$. Sie steigt fjordeinwärts z. T. bis auf $800 \mathrm{~m}$, und sinkt dann ostwärts rasch ab. Auf den unterkühlten polaren Gletschern liegt die lokale Schneegrenze, wie sie in der Firnlinie deutlich wird, wesentlich tiefer bei durchschnittlich 300-400 m Höhe.

Der W-Teil von Nathorst-Land weist nur einzelne Kar- und Talgletscher auf. Der E-Teil wird dagegen von einer mächtigen zusammenhängenden Vergletscherung eingenommen. Dieses Inlandeis fließt ab mit mächtigen Gletschern zu den Enden der Fjorde, wo hohe Gletscherfronten zum Meer abbrechen.

Große Alt-Moränen aus gestauchten marinen Sanden reichen weit fjordauswärts, rd. $35 \mathrm{~km}$ am Paulabreen im N und rd. $25 \mathrm{~km}$ am Nathorstbreen im S. Diese Alt-Moränen zeigen, daß die großen Gletscher der holozänen Vereisung erhebliche Schwankungen erlebt haben. Ahnliche, jedoch geringere Schwankungsspuren findet man am Fridtjovbreen und am Recherchebreen.

Die kleineren Talgletscher zeigen außer den jüngsten Endmoränenloben keine älteren vorgeschobenen Moränenkränze. Die holozänen marinen Terrassen sind nur an wenigen Stellen, z. B. am Penckbreen, vor den Moränen aufgestaucht. Man muß annehmen, daß diese Gletscher seit der postglazialen Wärmezeit nicht über das Maximalstadium, das ungefähr der zweiten Hälfte des 19. Jh. entsprechen dürfte, hinaus vorgestoßen sind.

Das Wedel-Jarlsberg-Land hat mächtige nur z. T. isolierte Gletscherzungen als Abfluß eines inneren Vereisungsnetzes. Nur der Recherche- und der Renard-Breen stoßen unmittelbar bis zum Meer vor. Das Nordenskiöld-Land ist auffallend wenig vergletschert wegen der lokal hohen Lage der Schneegrenze um den Eis-Fjord. Nur die E-Flanke des westlichen Gebirgsrandes schickt einen größeren Gletscher nach N zum Grön-Fjord und nach $\mathrm{S}$ den Fridtjov-Breen.

\section{Die pleistozäne Vereisung}

Die weiten Taltröge sind vom pleistozänen Eisstromnetz geprägte Vorzeitformen. Nur die Hangentwicklung und die Flußterrassen sind Zeugen holozäner rezenter Überformung. Soweit das Eis im Pleistozän die Berge überflossen hat, wurden sie zu Kuppenformen zugeschliffen. Im Davisdalen am Van-Keulen-Fjord wird man aufgrund der Großformen 
annehmen müssen, daß die Vereisungsoberfläche sicher einmal oberhalb der Bergkuppen gelegen hat, also höher als $700 \mathrm{~m}$. Eine oberste Schliffgrenze ist jedenfalls hier nicht ausgebildet.

Anders ist es bei den höheren Bergspitzen des Midterhuken (782 m) und des Berzeliustinden $(1205 \mathrm{~m})$. Diese Gipfel sind auch bei der pleistozänen Vereisung nicht überflossen worden, sondern haben als Nunataker aus dem Eis geragt, ähnlich wie heute die kleinen Felsspitzen in den Gletscherflächen. Nur die Flanken der Berge am Bellsund sind vom Eis bis rd. $650 \mathrm{~m}$ Höhe überschliffen. Am schmalen Grat der $856 \mathrm{~m}$ hohen Basilika südlich des Van-Keulen-Fjords kann man die Schliffgrenze bei $\mathrm{rd} .760 \mathrm{~m}$ angeben (Abb. 2).

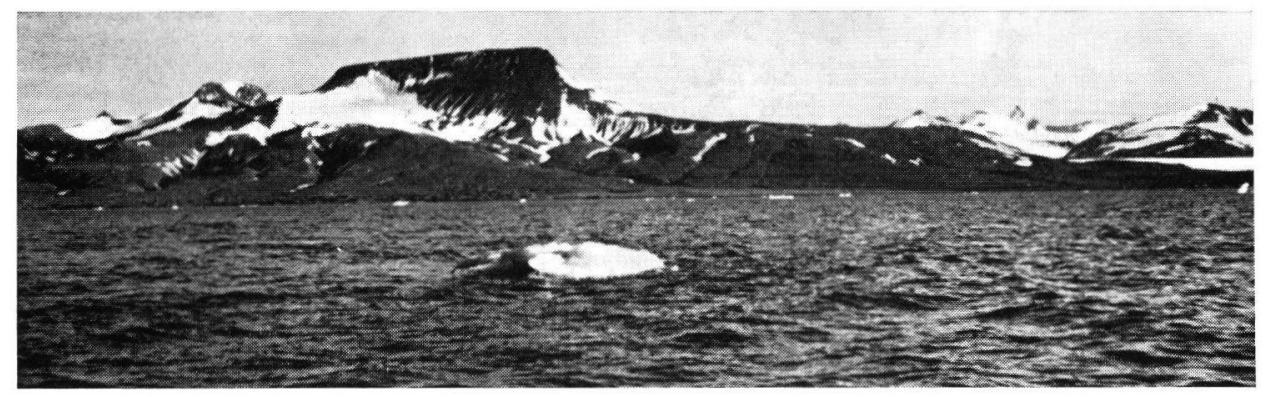

Abb. 2. Schliffgrenze bei rd. $760 \mathrm{~m}$ (oberhalb der schneegefüllten Runsen) an der Basilika (856 m) an der Südküste des Van-Keulen-Fjords.

Deutliche Gletscherschliffe sind im Bereich der oberen Schliffgrenze selten. Die Felsen an der Schliffgrenze, meist grobklüftiges Gestein, sind seit dem Rückgang der pleistozänen Vergletscherung der exzessiven physikalischen Verwitterung der Frostschuttzone ausgesetzt gewesen. Gletscherschliffe sind nur dort erhalten, wo sie im Trogbodenbereich überdeckt bis heute konserviert wurden. Haupt-Schliffstriemen zeigen in die Hauptrichtung des Fjord-Gletschers. Nebenrichtungen entsprechen lokalen Einflüssen von Seitengletschern, als sich im Spätglazial regionale Vereisungszentren ausgebildet hatten. Nur von den jung zurückweichenden Gletschern sind frische, deutliche Gletscherschliffe erhalten. Sie spiegeln aber nur die lokale holozäne Gletscherentwicklung wider.

Am westlichen Außenrand der pleistozänen Vereisung sind deutlich gletschergeschliffene Walfischrücken im Anstehenden der Vorlandplatte am Kap Lyell ausgeformt. Die Eisstoßrichtung von $\mathrm{E}$ her läßt sich noch erkennen. Sie sind aber bereits stark von der Frostverwitterung angegriffen. Die Eisbedeckung kann hier nicht mehr sehr mächtig gewesen sein, da hier Unterschiede des Untergrundes vom Eis zu solchen Formen potenziert wurden. Im inneren Fjordbereich fehlen solche vergleichbare Formen. Dort war die Eisüberdeckung offenbar so groß, daß Unebenheiten des Felsuntergrundes keine Differenzierung der Exaration verursachten.

Nach dem Verlauf der oberen Schliffgrenze ergibt sich folgendes Bild der pleistozänen Eismächtigkeit (Abb. 3): Im S des Arbeitsgebietes steigt die Vereisungsobergrenze von $400 \mathrm{~m}$ am Fjordausgang über $650 \mathrm{~m}$ am Berzelius-Tinden auf $760 \mathrm{~m}$ an der Basilika; im Nathorst-Land von $680 \mathrm{~m}$ am Midterhuken über $760 \mathrm{~m}$ am Sven-Nilsson-Fjellet bis auf über $800 \mathrm{~m}$ am Scheele-Fjellet; im südlichen Nordenskiöld-Land von $550 \mathrm{~m}$ am IngeborgFjellet über $780 \mathrm{~m}$ am Litledals-Fjellet bis rd. $800 \mathrm{~m}$ am Liljevalch-Fjellet.

Unsere Ergebnisse stimmen in der Größenordnung mit den Untersuchungen überein, die Нӧввом (1911) im westlichen Eis-Fjord durchführte und aufgrund deren er auf eine Eismächtigkeit bis $600 \mathrm{~m}$ geschlossen hat.

Die Fjorde sind deutlich glazial übertieft; der Van-Mijen-Fjord $177 \mathrm{~m}$ gegenüber der $60 \mathrm{~m}$ hohen Schwelle von Akselöya. Aufgrund des raschen Absinkens der pleistozänen 
NORDENSKIÖLDLAN D

Wsw

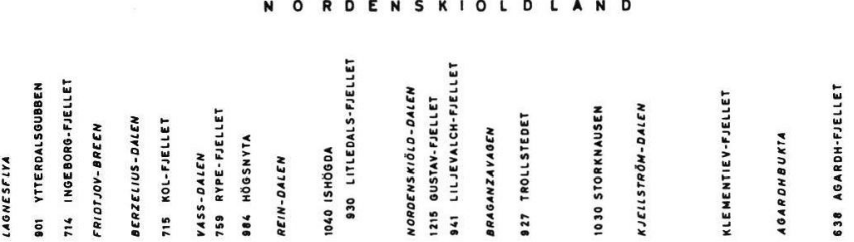

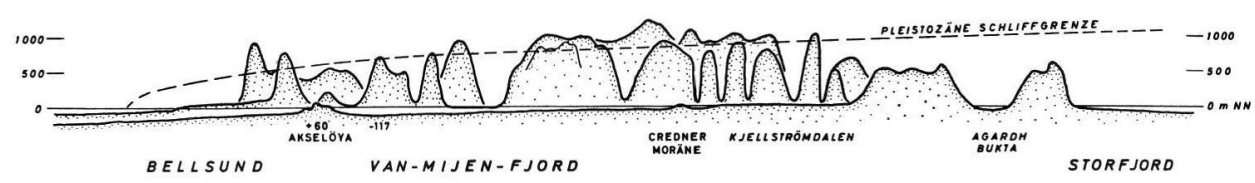

ro- rach übernônt

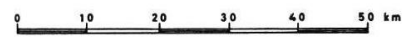

Enteurl u. Zeichnung: StäBleIN os

Abb. 3. Verlauf der pleistozänen Schliffgrenze im südlichen Nordenskiöld-Land.

Schliffgrenze nach W und den Erfahrungswerten über das Verhältnis von Eisdicke zu Eisrandentfernung muß man annehmen, daß das pleistozäne Eis nur wenige Kilometer über die Westküste hinausgereicht haben kann.

Das pleistozäne Eis ist nach $\mathrm{E}$ angestiegen, so daß man auf eine Eisüberfließung von einem östlich liegenden Vereisungszentrum her schließen kann. Diese Annahme wird noch durch den mächtigen Trog des Kjellströmdalen bestätigt, dessen Formung nur durch eine Gletscherbewegung vom Gebiet des Stor-Fjords her erklärt werden kann. Überregionale Erratika, die einen Vereisungszusammenhang nachweisen, konnten im Bellsundbereich nicht festgestellt werden. Dies ist aber nicht verwunderlich, da die tiefe Rinne des StorFjords von E her mitgeführtes Grundmoränenmaterial wahrscheinlich abgefangen hat.

De GeER hat schon 1900 den Zusammenhang der pleistozänen Vereisung der spitzbergischen Inseln nachzuweisen versucht. BüDEL (1960, S. 91 und Abb. 27) bestätigte den Eiszusammenhang mit einem Vereisungszentrum im E. Corbel (1960) und Schytт (1967) haben die Meinung vertreten, daß diese Barentseevereisung mit dem nordeuropäischen Eis zusammengeschlossen war, was SEMEvski (1967a) jedoch für unmöglich hält.

\section{Die marinen Terrassen und die Landhebung}

Die durchschnittlich $700 \mathrm{~m}$ mächtige, nach W abnehmende pleistozäne Vereisung hat nicht nur direkt durch die glazigene Formung auf die Morphologie eingewirkt, sondern wohl auch indirekt durch die gewaltige Eisbelastung und holozäne Entlastung eine glazialisostatische Landhebung verursacht, wie sie durch gehobene Strandlinien und marine Terrassen, Spuren alter Meeresstände, wahrscheinlich zu machen ist.

Die Theorie einer isostatischen Reaktion aufgrund pleistozäner Eisbelastung wurde als Erklärung für Landhebungen bereits 1865 von JAMIESON aufgestellt. NANSEN (1922) wendete diese Anschauung dann auf Spitzbergen an. $\mathrm{Ob}$ es sich dabei wirklich allein um eine echte Isostasie handelt, die bis in die Unterkruste der Erde oder gar bis in den Erdmantel, wie DALY (1934) meint, hineinreicht, erscheint von den heutigen geophysikalischen Anschauungen zweifelhaft. Eine glazigene Modifikation einer Schildaufwölbung, die älter ist als die pleistozänen Eisbelastungen, erscheint wahrscheinlicher (SEMEvski 1967). Die ${ }^{14} \mathrm{C}$-Datierung höherer mariner Terrassen $(84,5 \mathrm{~m})$ im Billefjord auf 21300 Jahre vor heute (FEYLING-HANSSEN 1965), also in eine Zeit in der die Eisbelastung noch wirkte, spricht für eine Hebungstendenz, die z. T. unabhängig ist von der Eisentlastung.

An der Südküste des Van-Mijen-Fjords liegen 8 Terrassen übereinander (Abb. 4). Die oberen steigen jedoch parallel zur geologischen Struktur an. Es sind also Strukturgesimse, die sich an Felszonen anlehnen. Nur die vier unteren Terrassen, die bis $60 \mathrm{~m}$ reichen, ver- 


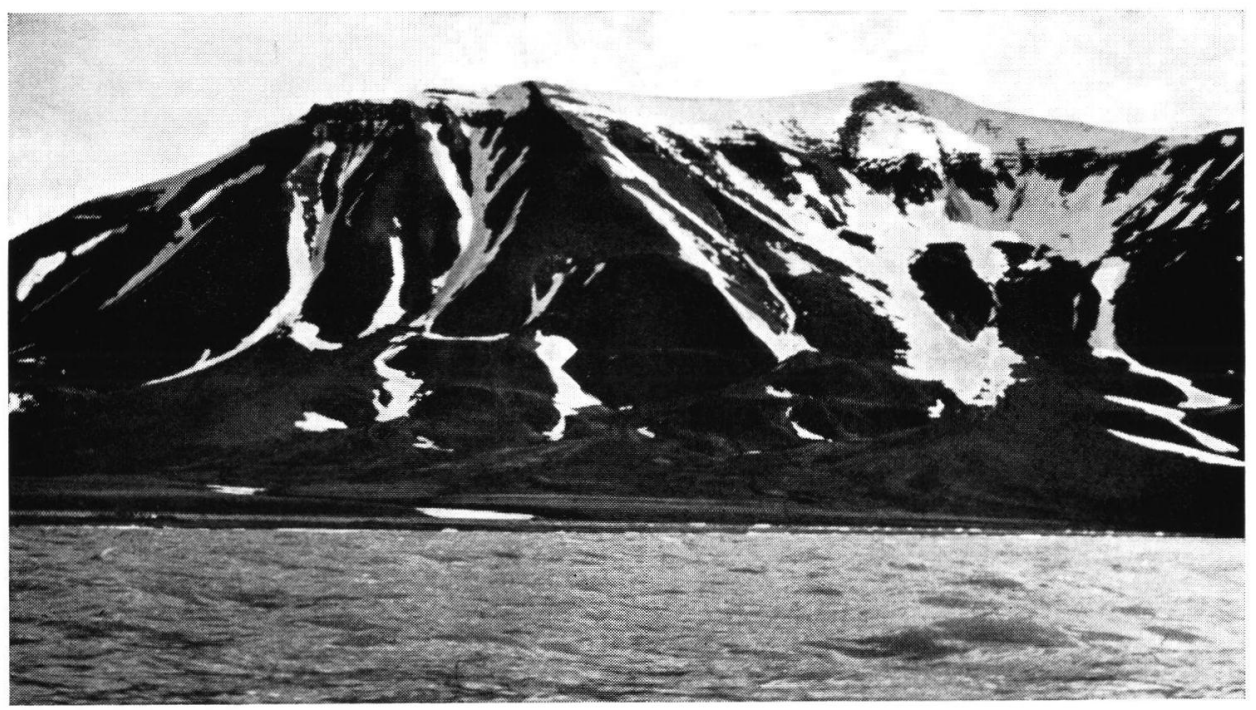

Abb. 4. Marine Terrassen und Struktur-Gesimse an der Südküste des Van-Mijen-Fjords.

laufen waagerecht, diskordant zur geologischen Struktur. Nur diese sind marine Terrassen. Inwieweit es sich bei den höheren Strukturgesimsen um akkordant als Schliffbord umgestaltete ältere Formen handelt, etwa um Eisrandlagen oder gar um vorglaziale Stufen, dafür haben sich keine konkreten Anhaltspunkte ergeben.

Marine Terrassen und Strukturterrassen kommen meist vermischt vor, so daß jeweils die speziellen geologischen Verhältnisse zu berücksichtigen sind. Im Bellsundbereich handelt es sich geologisch um einen flachen Sedimentationstrog mit Schichten des Mesozoikums bis herauf zum kohleführenden Tertiär über Plattformen des kambrischen Basements. Dieser Sedimentationstrog ist nach W zu entlang einem NNW-SSE-Lineament faltig aufgeschoben auf den kambro-silurischen, kaledonisch konsolidierten Hekla-Hoek-Komplex aus unterschiedlichen Sparagmiten.

Da fast alle Schichten Konglomerathorizonte enthalten, sind auch Gesimse oberhalb der marinen Grenze von Konglomeratgeröllen überstreut, die leicht mit holozänen marinen Strandgeröllen verwechselt werden können.

Das Profil der marinen Terrassen überblickt man z. B. an der Strandvollsletta. Bei den unteren Strandwallserien wird das verschiedene Alter auch farblich deutlich. Man kann eine weiße, grüne und braune Serie unterscheiden (Büdel 1962, S. 343). Die Strandwallserien steigen an bis zur 20-m-Terrasse, die im ganzen Gebiet auffallend breit ausgebildet ist. Sie entspricht vermutlich einer Ausgleichsphase von glazial-isostatischer Landhebung und glazial-eustatischem Meeresspiegelanstieg.

Über der 20-m-Terrasse folgen Terrassen-Reste in verschiedener Höhe bis $60 \mathrm{~m}$, die häufig durch ein fossiles Kliff abgeschlossen werden. In fast allen mittelgroßen Tälern treten um $86 \mathrm{~m}$ Höhe markante Terrassen-Riegel auf. In den großen Tälern fehlt der Riegel. Die Riegel bestehen aus großen Geröllen bis $20 \mathrm{~cm} \phi$ in einer sandigen Matrix. Marine Spuren ließen sich darin nirgends nachweisen. Es kann sich hier um glazial aufgestauchte Talfüllungen handeln, die vorher fluviatil delta-artig geschüttet wurden und nach der Stauchung von einem rd. 85-m-Meeresstand zu einer spätglazialen marinen Terrasse eingeebnet wurden. $\mathrm{Da}$ in den großen Tälern damals noch Gletscher mündeten, konnten sich dort keine Riegel-Terrassen ausbilden. Der marine Charakter der Terrassen bis $60 \mathrm{~m}$ Höhe ist durch Treibholz und Muscheln in den Terrassen-Sedimenten erwiesen. 


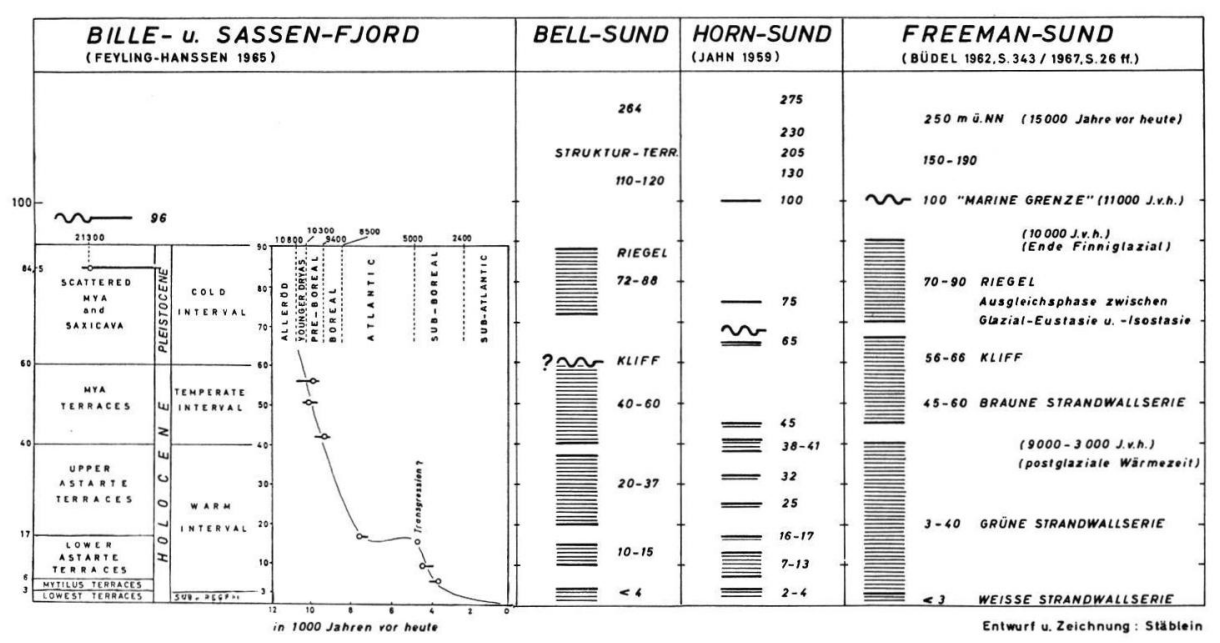

Abb. 5. Vergleich der Strandterrassen des Bellsund-Bereichs mit anderen Gebieten Spitzbergens.

Treibholz kann im heute baumlosen Spitzbergen nur vom Meer in das Sediment gebracht worden sein. Walknochen wurden nur auf der subrezenten Terrasse bis $4 \mathrm{~m}$ Höhe gefunden. Auch die Form der Terrassen-Sedimente (Brandungsgeröllpflaster) gibt Hinweise auf die marine Formung.

Zusammenfassend läßt sich über die holozäne Landhebung sagen, daß bei mindestens rd. $60 \mathrm{~m}$ vielleicht bei $85 \mathrm{~m}$ die obere marine Grenze im Bellsundbereich liegt. Höher am Hang als die marinen Stufen treten an vielen Stellen besonders deutlich am westlichen Fjordausgang in 120-100 $\mathrm{m}$ Höhe nach W zu abfallende, markante Stufen auf. Sie schneiden den Untergrund diskordant. In die Täler hinein steigen sie deutlich an. Stellenweise ist diese Stufe im Anstehenden als Schliffbord glazial überarbeitet und von Moränenmaterial überstreut. Es handelt sich in der heutigen Ausprägung um eine spätglaziale Eisranderscheinung aus der Zeit des Eisabbaus bei einer nur noch niedrigen regionalen Vergletscherung, also um eine zweite niedrigere Schliffgrenze.

\section{Eisbelastung und Landhebung}

Die marinen Terrassen steigen auf der $70 \mathrm{~km}$ langen Strecke des Bellsundbereichs ostwärts nicht an. Wie läßt sich erklären, daß trotz einer nach $\mathrm{W}$ abnehmenden Eisbelastung die marinen Terrassen keine unterschiedliche Landhebung widerspiegeln, während BüDEL (1968) für SE-Spitzbergen einen Anstieg der marinen Strandlinien nach E feststellte?

Eine Erklärung könnte die unterschiedliche Struktur der Inseln liefern. Während West-Spitzbergen mit dem kaledonisch konsolidierten Basement auf die Eisbelastung im Block oder in Blöcken reagiert hat, wurde der geologisch labilere Südosten entsprechend der unterschiedlichen Belastung verbogen. Diese Erklärung entspricht der Darstellung von Semevski (1967) des neotektonischen Zyklus von Spitzbergen als einer PlattformEntwicklung. $\mathrm{Zu}$ der Auffassung einer im-Block-Hebung von West-Spitzbergen passen auch die Terrassenhöhen in der Agardh-Bucht und bei Kvalvagen im Stor-Fjord, die GLASER (1968) festgestellt hat.

Der Prozeß der Landhebung scheint heute abgeschlossen $\mathrm{zu}$ sein, was durch junge Kliffbildungen nachgewiesen wird. Entsprechende Beobachtungen haben WiRTHMANN (1964) und Glaser (1968) bereits aus SE-Spitzbergen beschrieben.

Vergleichen wir unsere Ergebnisse noch mit denen Feyling-Hanssens (1965) aus dem Bille-Fjord und von JAHN (1959) aus dem Horn-Sund, sowie von BüDEL $(1962,1967)$ 
aus dem Freeman-Sund, so fügen sich die Befunde des Bellsund-Bereichs gut in diesen Rahmen (Abb. 5). Eine analoge Altersstellung erscheint wahrscheinlich. JAHN gibt ausdrücklich nur bis $65 \mathrm{~m}$ marine Terrassen an, während er die Stufen bei $75 \mathrm{~m}$, was unseren Riegelniveau entsprechen könnte, und höhere bis $275 \mathrm{~m}$ als alte Kliffs und Brandungsplatten mit Geröllagen bezeichnet. Diesen entsprechende Stufen haben wir im BellsundBereich als Struktur-Terrassen mit Konglomeratgeröllen nachgewiesen.

Die Landhebung ist im strengen Sinn keine einfache Isostasie, sondern die elastoplastische endogene Reaktion der Erdkruste auf die exogene Beanspruchung durch die quartäre Eisbelastung und Eisentlastung, die eine ältere Hebungstendenz modifizierend überlagert.

\section{Literatur}

BüDEL, J.: Die Frostschuttzone SE-Spitzbergens. - Colloquium Geographicum, 6, 105 S., Bonn 1960.

- : Die Abtragungsvorgänge auf Spitzbergen im Umkreis der Barentsinsel auf Grund der Stauferland-Expedition 1959/60. - Verh. 33. Deutsch. Geogr. Tag. Köln 1961, 337-375, Wiesbaden 1962 .

— : Die Abtragungsvorgänge in der exzessiven Talbildungszone Südost-Spitzbergens. (Vorläufiges Manuskript für: Ergebnisse der Stauferland-Expedition 1959/60, 1, Wiesbaden. An alle Teilnehmer der Stauferland-Expedition 1967 im Manuskript-Abdruck verteilt.)

- : Die junge Landhebung Spitzbergens im Umkreis des Freeman-Sundes und der Olgastraße. Würzburger Geogr. Arb., 22/I, 21 S., Würzburg 1968.

Conbel, J.: Le soulèvement des terres autour de la mer de Barents. - Revue de Géogr. de Lyon, 35, 3, 253-274, Lyon 1960.

DaLY, R.: The changing world of the ice age. - New Haven 1934.

DE Geer, G.: Om östra Spetsbergens glaciation under istiden. - Geol. För. Förh., 22, Stockholm 1900.

Feyling-Hanssen, R. W.: Shoreline displacement in central Spitsbergen. - Ergebnisse der Stauferland-Expedition 1959/60, 3, Vorträge des Fridtjov-Nansen-Gedächtnis-Symposions über Spitzbergen 1961 in Würzburg, 24-28, Wiesbaden 1965.

GlaSER, U.: Junge Landhebung im Umkreis des Storfjord (SO-Spitzbergen). - Würzburger Geogr. Arb., 22/II, 22 S., Würzburg 1968.

Нӧявом, B.: Bidrag till Isfjordsomradets Kvartärgeologie. - Geol. För. Förh., 23, Stockholm 1911.

JAHN, A.: The raised shorelines and beaches in Hornsund and the problem of postglacial vertical movements of Spitzbergen. - Przeglad Geograficzny, 31, Suppl., 143-178, Warschau 1959.

- : Postglacjalny rozwoj wybrzezy Spitzbergebu. (Postglaziale Entwicklung der Küsten Spitzbergens.) - Czasopismo Geograficzne, 30, 3, 245-262, Warschau 1959.

Jamieson, T. F.: On the history of the last geological changes in Scotland. - Quart. J. geol. Soc., 16, London 1865.

Nansen, F.: The Strandflat and Isostasy. - Vidensk. Selsk. Skrifter, 1, 11, 313 S., Oslo 1922.

Schytt, V., Hoppe, G., Blake, W. u. Grosswald, M. G.: The extent of the Würm Glaciation in the European Arctic; a preliminary report about the Stockholm University Svalbard Expedition 1966. - International Union of Geodesy and Geophysics (IUGG), General Assembly of Bern 25. 9.-7. 10. 1967, Commission of Snow and Ice, Reports and Discussions, Publication 79, 207-216.

Semevski, D. W.: Neotektonik des Spitzbergen-Archipels (russisch). In: Material über die Stratigraphie von Spitzbergen, hrsg. v. Inst. f. Geologie der Arktis, 225-238, Leningrad 1967.

- : Die Hauptstufen der Entwicklung Spitzbergens während Pliozän und Quartär (russisch). In: Material über dic Stratigraphic von Spitzbergen, hrsg. v. Inst. f. Geologic der Arktis, Lenin$\operatorname{grad} 1967$ [1967a].

Wirthmann, A.: Die Landformen der Edge-Insel in Südost-Spitzbergen. - Ergebnisse der Stauferland-Expedition 1959/60, 2, 66 S., Wiesbaden 1964.

Manuskr. eingeg. 30. 6. 1969.

Anschrift des Verf.: Dr. Gerhard Stäblein, wiss. Assistent am Geographischen Institut der Universität Würzburg, 87 Würzburg, Klinikstraße 3. 